\title{
Understanding and Managing Spontaneous Intracranial Hypotension
}

Can J Neurol Sci. 2013; 40: 139-140

There are few clinical problems in clinical neurology, that are more interesting to diagnose and manage than headache as a result of spontaneous intracranial hypotension $(\mathrm{SIH})$. The early identification of a cerebral spinal fluid (CSF) leak with appropriate management can lead to quick and gratifying results for the patient and the treating clinicians. Otherwise, initial and ongoing failures to find the CSF leak in a patient with classical symptomology and signs of SIH, clinically and radiological, can direct the patient and their physicians down a long and winding road in order to find the putative leak and repair it successfully.

Spontaneous intracranial hypotension is becoming a more commonly recognized cause of headache with a spectrum of presentations, classically presenting with low CSF pressure and magnetic resonance enhancement of the pachymeninges or the dura $^{1,2}$. The classical headache is frequently holocephalic and bilateral and positional in nature, worse with being upright, however not all orthostatic headaches are due to $\mathrm{SIH}^{1,2}$. These patients need detailed investigation and referral for expert opinion given the difficulty in finding the CSF leak and treating it given the complexity and clinical variability.

Positional changes are very important, but not essential in all cases and some cases are paradoxical in that the headache is better standing than lying ${ }^{3}$, or they have chronic headache without position change. Some patients have the syndrome without pachymeningeal enhancement ${ }^{4}$ or the enhancement without the headache ${ }^{5}$. Other clinical features include: diplopia, blurring of vision, or visual field change. Cranial nerve abnormalities are rare and patients may complain of facial numbness, dizziness, and interscapular, neck low back pain ${ }^{1,2}$. Encephalopathy or altered states of consciousness have been reported ${ }^{6}$.

In this volume of the Journal there is an excellent review by the Calgary group on the management of $\mathrm{SIH}^{7}$. This article is comprehensive in its breadth and description of the disorder, including a detailed analysis of the various diagnostic modalities required to make the diagnosis in appropriate patients, many of who have complex histories. This is a disorder with uncertain pathophysiology; one which defies simple explanation in many cases and possibly this is because of its syndromic nature or perhaps it can have more than one pathophysiological mechanism participating in the production of the symptomology, or several etiologies, including the association with connective tissue disorders.

The authors are to be congratulated on their thorough review of the subject, the diagnostic criteria, discussion of etiopathogenesis and investigations, along with an overview of multiple therapeutic interventions. Not only do they provide an excellent review of the literature and discussion, but also in tables and algorithms they outline a most reasonable approach to this disorder.

A multiple disciplinary approach is required in most instances to diagnose and manage patients with SIH. The authorship of this paper includes not only a neurologist with expertise in headache medicine, but also an anesthesiologist and neuroradiologists ${ }^{7}$. It is valuable to have a team of the right people, with knowledge of the variability of presentations of this disorder, working together to find the best management strategy for each individual patient. The authors are correct in pointing out that there is no large randomized trials on the treatment of this disorder that show a consistent approach, and most of the time SIH cases are dealt individually in different centers by different specialists. Thus this review allows some statement of consistency that is an entirely reasonable approach, I believe, at present.

Our meninges are remarkable in assisting our brains to float inside our heads, and particularly it is the dura mater that seals the balloon and allows us to walk in the upright position on this remarkable CSF cushion. When there is some deficiency of dural integrity either due to direct puncture, leakage or failure of the anatomical nature of the dura mater itself to contain the CSF then the result becomes symptomatic in most patients. Thus it would be somewhat reasonable to consider SIH as a disorder of 'dural insufficiency', which directly or indirectly results in the downward sagging of the intracranial structures into the posterior fossa and foramen magnum with effects at the root levels in the spinal cord. When this happens headache is the major manifestation presenting with a postural component; yet there are numerous other neurological symptoms that can occur in $\mathrm{SIH}$ as are outlined in the review.

Thus our brains are protected by a floating mechanism with mechanics that obey to a large extent the laws of physics as best they can be applied to biological systems ${ }^{8}$. It is surprising to me that there are not more CSF leaks in human beings given our activities of everyday life, the high incidence of major and minor traumas we inflict upon ourselves and other insults to our brains and spinal cords.

Treatment can be difficult and ranges from conservative to invasive surgical management strategies ${ }^{1,2,7}$. The concept of using non-directed epidural blood patches following initial imaging confirmation of the diagnosis of SIH deserves more attention ${ }^{1,2,7}$. Directed epidural blood patches are probably the best treatment if the sight if the leak can be localized ${ }^{1,2,7}$. More specialized invasive surgical options should only be reserved for the most intractable cases and probably should be utilized with great care $^{1,2,7}$.

I highly recommend this comprehensive review to all who are involved in the care of patients with SIH. It is our job to find and repair the dura and restore its integrity or at least start the biological processes of healing.

R. Allan Purdy Dalhousie University, Halifax, Nova Scotia, Canada 


\section{REFERENCES}

1. Schievink WI. Spontaneous spinal cerebrospinal fluid leaks and intracranial hypotension. JAMA. 2006;295(19):2286-96.

2. Wang YF, Wang SJ. Headache associated with low CSF pressure. Medlink Neurology. Available from: www.medlink.com. April 2012.

3. Mokri B, Aksamit AJ, Atkinson JL. Paradoxical postural headaches in cerebrospinal fluid leaks. Cephalalgia. 2004;24(10):883-7.

4. Mokri B, Atkinson JLD, Dodick DW, Miller GM, Piepgras DG. Absent pachymeningeal gadolinium enhancement on cranial MRI despite symptomatic CSF leak. Neurology. 1999;53:402-4.
5. Mokri B, Atkinson JLD, Piepgras DG. Absent headaches despite CSF volume depletion (intracranial hypotension). Neurology. 2000;55:1722-4.

6. Ghavanini AA, Scott CA, Chan DK, Tang-Wai DF. Management of patients with spontaneous intracranial hypotension causing altered level of consciousness: Report of two cases and review of literature. Cephalagia. Epub 2012 Nov 9.

7. Amoozegar F, Guglielmin D, Hu W, Chan D, Becker WJ, Spontaneous intracranial hypotension: recommendations for management. Can J Neurol Sci. 2013;40(2):144-57.

8. Mokri B. The Monro-Kellie hypothesis. Applications in CSF volume depletion. Neurology. 2001;56:1746-8. 